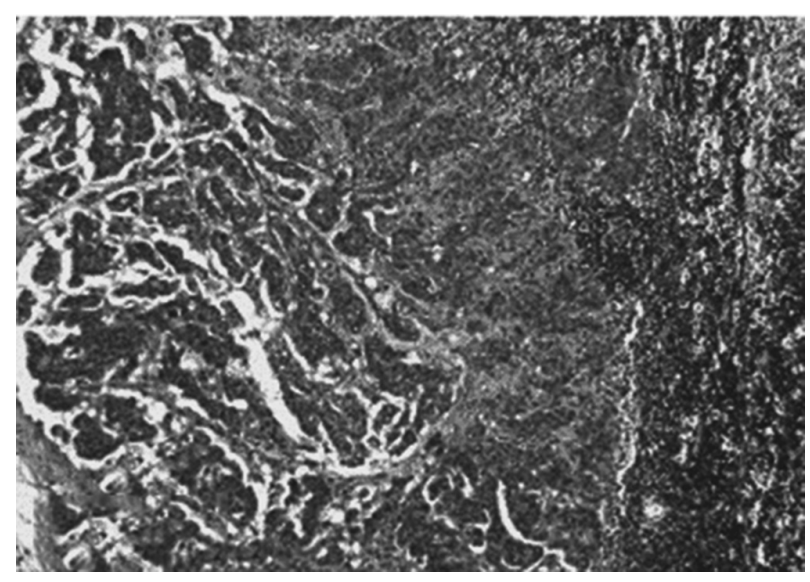

Abstract IDDF2019-ABS-0055 Figure 4

increasingly severe and unbearable. But no obvious abnormality was found in physical examination except varicose veins in the lower extremities. His gastroscope found severe gastric varices with a red-color sign and no active bleeding (figure 1.). So what causes the Severe gastric varices? For the isolated gastric varices (IGV), he was first suspected of liver cirrhosis, but he had no medical history of viral hepatitis, and the liver fibrosis determination was normal. Laboratory findings were as follows: white blood cells:5.11*10\%/L; hemoglobin: $156 \mathrm{~g} / \mathrm{L} ; \quad$ platelets: $180 * 10^{9} / \mathrm{L} ; \quad \mathrm{CEA}: 185 \mathrm{ng} / \mathrm{ml}$ and CA199:1000.00U/ml. Due to the increase of CA199 and no evidence of liver cirrhosis, we did more examinations. The investigation revealed multiple low-density foci occur in the pancreas on computed axial tomography(CT) of the portal vein (figure 2.). These low-density foci are multiple cystic lesions of the pancreas, with the largest lesions about $1.6 \mathrm{~cm}$ in diameter.

Results This patient was taken to the operation. After laparotomy, a $4 * 5 \mathrm{~cm}$ tumor was found in the body of the pancreas (figure 3.). The tumor was hard, adherent to the surrounding tissues, and the para-aortic lymph nodes were enlarged. The general surgical team resection the para-aortic lymph nodes and the freezing results are reported as adenocarcinoma. So they decided to perform distal pancreatectomy and splenectomy. After the operation, the specimens were sent to the pathology examination to be confirmed as moderately/poorly differentiated pancreatic ductal adenocarcinoma, and partly mucinous adenocarcinoma (figure 4.).

Conclusions So we should pay attention to the possibility that IGV may be caused by pancreatic lesions, as well as the possibility of malignant transformation of PCN.

\section{IDDF2019-ABS-0056 MINIMALLY INVASIVE ESOPHAGECTOMY AFTER NEOADJUVANT CHEMORADIOTHERAPY USING CROSS REGIMEN FOR LOCALLY ADVANCED ESOPHAGEAL CANCER}

Kalayarasan Raja*. JIPMER, India

\subsection{6/gutjnl-2019-IDDFabstracts. 130}

Background Neoadjuvant therapy followed by surgery is the current recommended treatment for esophageal cancer. Since the publication of the landmark trial on neoadjuvant chemoradiotherapy (NACTRT), CROSS regimen has been commonly used for locally advanced esophageal cancer. However, the safety of CROSS regimen; the feasibility and shortterm outcomes of minimally invasive esophagectomy (MIE) with total mediastinal lymphadenectomy after CROSS regimen has not been studied in Indian population where squamous cell carcinoma remains the predominant histological subtype and presentation with locally advanced tumor and severe malnutrition is common.

Methods A retrospective analysis of a prospectively collected database of patients with histologically proven esophageal cancer and eligible for NACTRT (cT1-3, N0-1, M0). All patients underwent thoracoscopy or robot-assisted MIE with total mediastinal lymphadenectomy including dissection along the bilateral recurrent laryngeal nerve. The parameters analyzed were clinicopathological features, dropout rate with NACTRT, postoperative complications, and pathological response rate. The pathological response rate was analyzed by TRG (Tumor Regression Grading) system.

Results During the study period between August 2013 and March 2018, a total of 150 patients with esophageal cancer were admitted and evaluated. Of the 150 patients, 67 (44.6\%) were eligible for NACTRT by CROSS protocol. Of these, $37(55.2 \%)$ patients completed NACTRT followed by surgery. Thirty $(44.7 \%)$ patients did not complete NACTRT due to complications or poor compliance. Of the patients who underwent surgery majority underwent thoracoscopic assisted MIE $(n=33,89.1 \%)$ and four patients underwent Robotic-assisted MIE. Most common postoperative complication was pneumonia $(n=14,37.8 \%)$ followed by hoarseness secondary to recurrent laryngeal nerve palsy $(\mathrm{n}=12,32.4 \%)$. Complete pathological response (TRG 1 and 2) of the primary tumor was observed in 20 patients $(55.5 \%)$ and 11 patients $(30.5 \%)$ had a partial response (TRG 3 and 4). Of the 20 patients with the complete pathological response of the primary tumor $5(25 \%)$ had a node-positive disease.

Conclusions NACTRT followed by MIE is feasible in patients with locally advanced esophageal cancer. The nodal disease is common even in patients with the complete pathological response of the primary tumor. The dropout rate with NACTRT is high in Indian population.

\section{IDDF2019-ABS-0057 THE CLINICAL FOLLOW-UP ANALYSIS OF 127 PANCREATIC CANCERS PATIENTS}

Huang LiYa*. Department of Gastroenterology, the General Hospital of NinXia Medical University, Yinchuan, China

\subsection{6/gutjnl-2019-IDDFabstracts.131}

Background Pancreatic cancer (PC) is one of the most lethal malignant diseases with the worst prognosis. This study was to observe the PC patients clinical characters and followedup $\underline{3}$ years, to discuss the factors that affect the prognosis of PC.

Methods The study was retrospective of $127 \mathrm{PC}$ patients and followed-up $\underline{3}$ years. The data included the gender, age, PC family history, the onset symptoms, cancer metastasized and treatment.

Results The PC patients mean survival were 10.54 \pm 8.53 months and 3-year survival rate was $3.14 \%$. The stage of tumor, the serum of CA199 were main factors of survival. 